EPJ Web of Conferences 70, 00045 (2014)

DOI: 10.1051/epjconf/ 20147000045

(C) Owned by the authors, published by EDP Sciences, 2014

\title{
Highlights from the OPERA experiment
}

\author{
N. Mauria on behalf of the OPERA Collaboration \\ ${ }^{1}$ INFN - Laboratori Nazionali di Frascati, Via E. Fermi 40, I-00044 Frascati (Roma), Italy
}

\begin{abstract}
The OPERA neutrino experiment is designed to detect $v_{\mu} \rightarrow v_{\tau}$ oscillations in direct appearance mode. The hybrid apparatus consists of an emulsion/lead target complemented by electronic detectors. It is placed in the long-baseline CERN to Gran Sasso neutrino beam (CNGS) $730 \mathrm{~km}$ away from the source. The running of the experiment and the extraction of data recorded in the emulsion will be described, together with the special procedures used to locate interaction vertices and detect short decay topologies. New oscillation search results with increased statistics will be presented. Up-to-date results of the neutrino velocity measurements will also be reported.
\end{abstract}

\section{Introduction}

OPERA [1] is a long baseline neutrino experiment located in the underground Gran Sasso Laboratory (LNGS). It is aimed at detecting for the first time the appearance of tau neutrinos from the oscillation of muon neutrinos in the CERN to Gran Sasso beam-line. The direct appearance search is based on the event-by-event detection of $\tau$ leptons produced in Charged Current (CC) $v_{\tau}$ interactions. The topological observation of the short-lived $\tau$ decay requires an exceptional granularity integrated in a large mass. This is achieved in OPERA using Emulsion Cloud Chambers (ECC) and real-time electronic detectors with a modular structure.

In addition to its main goal, the experiment is well suited to search for the subdominant $v_{\mu} \rightarrow v_{e}$ oscillations and to determine the neutrino velocity with high accuracy through the measurement of the time of flight and of the distance between CERN and OPERA.

\subsection{The OPERA detector}

The ECC basic unit in OPERA is the so-called "brick", consisting of 56 lead plates, $1 \mathrm{~mm}$ thick, interspaced with 57 nuclear emulsion films acting as high accuracy $(O(\mu \mathrm{m}))$ tracking devices. Each brick weighs $8.3 \mathrm{~kg}$, the overall target is composed of about 150000 bricks for a total mass of $1.25 \mathrm{kton}$. The brick acts as a stand-alone detector for momentum measurement through Multiple Coulomb scattering [2] and for electromagnetic shower reconstruction, given its thickness along the beam direction (10 radiation lengths). The submicrometer spatial resolution of the nuclear emulsion allows a precise three-dimensional reconstruction of the neutrino vertex as well as of the decay vertex associated with short-lived particles, like the $\tau$ lepton.

ae-mail: mauri@lnf.infn.it

This is an Open Access article distributed under the terms of the Creative Commons Attribution License 2.0, which permits unrestricted use, distribution, and reproduction in any medium, provided the original work is properly cited. 


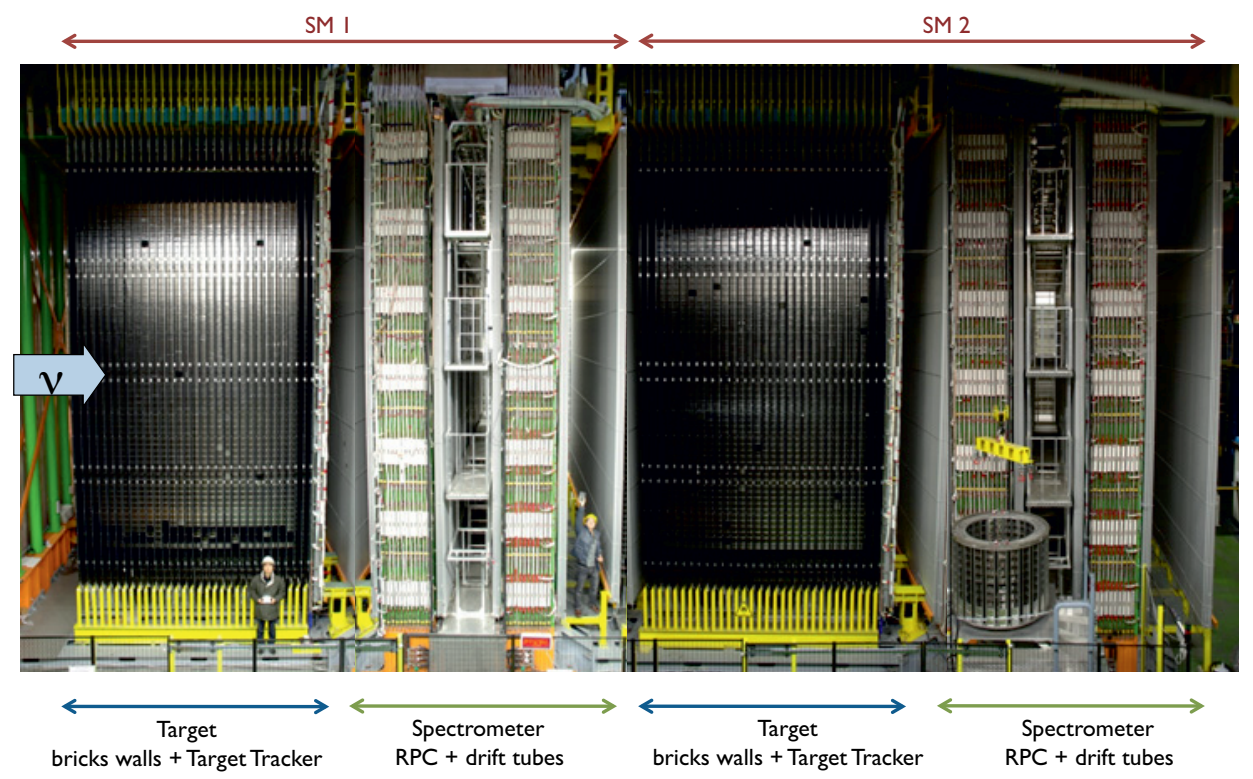

Figure 1. The OPERA detector in the Hall C at LNGS.

OPERA is a hybrid detector made of two identical Super Modules (SM) each consisting of a target section and a muon spectrometer, shown in Fig. 1. In the target, the bricks are arranged in 29 vertical "walls", transverse to the beam direction, interleaved with scintillator Target Tracker planes (TT) to trigger the read-out and localize the brick containing the neutrino interaction.

Tightly packed removable doublets of emulsion films called Changeable Sheets (CS) [3] are attached downstream of each brick in a separate envelope. They serve as interfaces between the TT and the brick, in order in order to validate its selection as containing the neutrino interaction and to drive with a precise prediction the location procedure in the ECC.

The target section is followed by a dipolar iron magnet instrumented with RPC and drift tube detectors, in order to measure the muon charge and momentum.

\subsection{The CNGS neutrino beam}

OPERA is exposed to the long baseline CNGS $v_{\mu}$ beam, $730 \mathrm{~km}$ away from the source. The beam is optimized for $v_{\tau}$ appearance search. The neutrino energy spectrum $\left(\left\langle E_{v_{\mu}}\right\rangle=17 \mathrm{GeV}\right)$ was designed to maximize the number of $v_{\tau}$ CC interactions, i.e. the convolution of CC interaction cross section (favoured at high energy) and oscillation probability (favoured at low energy, $\sim 1.5 \mathrm{GeV}$ ). In terms of interactions, the $\bar{v}_{\mu}$ contamination is $2.1 \%$, the $v_{e}$ and $\bar{v}_{e}$ contaminations are together smaller than $1 \%$, while the number of prompt $v_{\tau}$ is negligible.

The CNGS beam is produced by a $400 \mathrm{GeV} / \mathrm{c}$ proton beam extracted from the CERN SPS. During a nominal CNGS cycle $(6 \mathrm{~s})$, there are two SPS extractions separated by $50 \mathrm{~ms}$, with $10.5 \mu \mathrm{s}$ pulse duration.

At the end of the 2011 Run, the CNGS had accumulated $14.2 \times 10^{19}$ protons on target (pot). By the time of this conference (June 2012), about $15 \times 10^{19}$ pot were delivered. The numbers of pot and 
Table 1. CNGS performances and corresponding neutrino interactions in the OPERA detector: status and outlook.

\begin{tabular}{|c|c|c|c|}
\hline Year & Protons on target $($ pot $)$ & $v$ interactions in the bricks & integrated pot/proposal value \\
\hline 2008 & $1.78 \times 10^{19}$ & 1698 & $7.9 \%$ \\
2009 & $3.52 \times 10^{19}$ & 3557 & $23.6 \%$ \\
2010 & $4.04 \times 10^{19}$ & 3912 & $41.5 \%$ \\
2011 & $4.84 \times 10^{19}$ & 4210 & $63.0 \%$ \\
2012 & $\left(\sim 4.7 \times 10^{19}\right)$ & $(\sim 4050)$ & $(\sim 84 \%)$ \\
\hline
\end{tabular}

neutrino events in OPERA year-by-year are shown in Tab. 1. If the CNGS performance in 2012 will be in line with 2011 OPERA expects to complete the data taking having accumulated $18.9 \times 10^{19}$ pot, about $84 \%$ of the nominal design luminosity.

\section{Oscillation results}

OPERA is currently in its fifth year of data taking. The results here presented are based on the analysis of the first two years' total sample (2008-2009), which is completed [4], and of a 2010-2011 data sub-sample. The strategy currently followed for 2010-2012 data relies on the preselection of a signal-enriched sample obtained with the electronic detectors. Charged current interactions with low momentum negative muons $\left(p_{\mu^{-}}<15 \mathrm{GeV} / \mathrm{c}\right)$ and NC-like interactions are prioritized for 2010 data, while for 2011-2012 data the priority is given only to NC-like events.

\subsection{Detection of neutrino interactions}

The electronic detector triggers recorded on-time with the CNGS are divided by an offline algorithm in "external" events, i.e. mostly muons produced by $v_{\mu} \mathrm{CC}$ interactions in materials preceding OPERA in the beam line, and "internal" events, i.e. interactions occurring inside the OPERA target. For oscillation studies, only internal events are analysed, while external events are discarded. The automatic algorithm further classifies internal events into $\mathrm{CC}$ and $\mathrm{NC}$ interactions.

A dedicated program reconstructs the event tracks and builds a probability map for bricks to contain the neutrino vertex. The brick with the highest probability is extracted from the target, the CS films are detached, developed and analysed with high-speed automatic optical microscopes [5], searching for tracks compatible with the TT data to verify the brick selection. In case this search is unsuccessful, the brick is equipped with a fresh CS and inserted back into the target, while a second brick is extracted according to the probability map. In case of positive CS result the brick is unpacked and the emulsion films are developed and dispatched to one of the scanning laboratories in Europe and Japan.

The tracks found in the CS doublet are extrapolated to the most downstream film of the brick and followed upstream with a prediction scanning, until they are not found in three consecutive films, i.e. the stopping point is reached. Vertex confirmation is obtained by scanning a $\sim 2 \mathrm{~cm}^{3}$ volume around the stopping point. 


\section{$2.2 v_{\mu} \rightarrow v_{\tau}$ search}

The final phase of the event analysis is the decay search procedure. This procedure was developed to detect charged and neutral decay topologies, secondary interactions and electromagnetic showers in the neighborhood of the primary vertex.

When a secondary vertex is found the kinematical analysis of the whole event is performed. This analysis integrates the complementary information provided by emulsions and electronic detectors, making use of the angles measured in the emulsion films, the momenta determined by multiple Coulomb scattering measured in the brick, the momenta measured by the magnetic spectrometers, and the total energy deposited in the instrumented target acting as a calorimeter $[4,6]$. The energy of $\gamma$-rays and electrons is estimated by a Neural Network algorithm.

By applying this procedure, the first $v_{\tau}$ candidate event was observed in the decay search of 20082009 data, as reported in detail in Ref. [7]. The tau-candidate event has 7 prongs at the primary vertex, out of which 4 are identified as originating from a hadron and 3 have a probability lower than $0.1 \%$ of being caused by a muon, none being left by an electron. The parent track exhibits a kink topology and the daughter track is identified as produced by a hadron through its interaction. Its impact parameter with respect to the primary vertex is $(55 \pm 4) \mu \mathrm{m}$; the impact parameter is smaller than $7 \mu \mathrm{m}$ for the other tracks. Two $\gamma$-rays point to the secondary vertex. The event passes all the selection cuts defined in the experiment proposal. The invariant mass of the two $\gamma$-rays is $(120 \pm 20$ (stat.) \pm 35 (syst.) $\mathrm{MeV} / \mathrm{c}^{2}$, consistent with the $\pi^{0}$ mass. Together with the secondary hadron assumed to be a $\pi^{-}$they have an invariant mass of $\left(640_{-80}^{+125} \text { (stat. }\right)_{-90}^{+100}$ (syst.) $) \mathrm{MeV} / \mathrm{c}^{2}$. The decay mode is therefore compatible with being $\tau \rightarrow v_{\tau} \rho^{-}\left(\rightarrow \pi^{-} \pi^{0}\right)$, whose branching ratio is about $25 \%$.

Since the publication of the first $v_{\tau}$ candidate in 2010 several improvements have been introduced in the analysis:

- a new Monte Carlo framework integrating emulsion and electronic detector data; it allows a detailed simulation of all the steps of the reconstruction process, from brick location to decay search;

- automatic tools performing a systematic search for highly ionizing tracks in a wide field of view $\left(2.5 \times 2.1 \mathrm{~mm}^{2}\right)$; the tagging of nuclear fragments allows to better distinguish genuine decays from hadron reinteractions, resulting in a background reduction in the $\tau \rightarrow h$ channel of about 20\%;

- a new procedure, the "track follow-down", to follow the vertex tracks in the downstream direction using data from several bricks; profiting of correlations between momentum and range it is possible to obtain an increased $\mu$ identification efficiency, resulting in a reduction of background from both charm and mis-identified hadronic tracks from $v_{\mu}^{C C}$ interactions;

- the state-of-the art charm cross section from the CHORUS [8] experiment, which implies an increase of the charm background by a factor 1.6 to 2.4 depending on the decay channel, the main contribution coming from $D^{+}$decays.

Data driven hadron background constraints are being pursued:

- $v_{\mu}$ interactions with charm production have been studied. The charm sample offers the opportunity to benchmark the $\tau$ efficiency thanks to the similarity of the lifetime and the decay topologies. The observed events for the analysed sample (49 events) are compared to the full Monte Carlo expectation $(51.0 \pm 7.5$ events). The comparisons between data and Monte Carlo for the angle between the muon and the hadronic system in the transverse plane, called $\phi$-angle, for the charmed meson decay length and for the impact parameter of the charmed daughter are shown in Fig. 2.

- Hadronic tracks from CNGS neutrino interactions have been followed for a total length of $14 \mathrm{~m}$ in lead, which is equivalent to a sample of $2300 \mathrm{NC}_{\text {events }}{ }^{1}$. No events are found in the signal

\footnotetext{
${ }^{1}$ To mimic a $\tau$ decay the interaction should happen within the first two lead plates.
} 

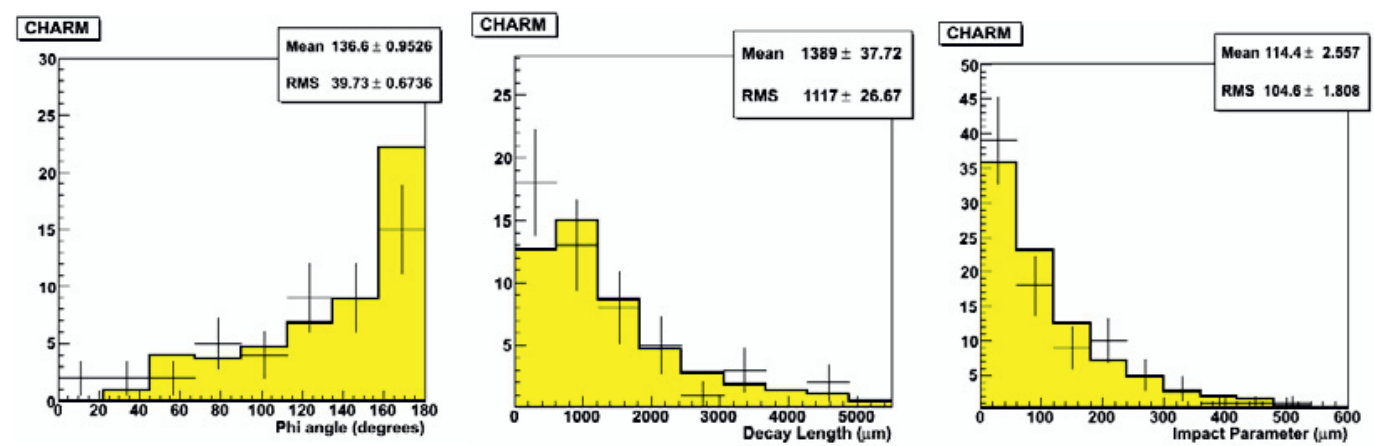

Figure 2. From left to right, experimental data (black error bars) superimposed to Monte Carlo data (yellow histogram): a) Distribution of the angle $\phi$ between the primary muon and the hadronic system in the transverse plane. b) Distribution of the decay length of the charmed meson. c) Distribution of the impact parameter of the charmed meson with respect to the primary vertex.

region defined by requiring 1-prong hadronic interactions with $\theta_{\text {kink }}>20 \mathrm{mrad}, p>2 \mathrm{GeV} / \mathrm{c}$ and $p_{T}>0.6 \mathrm{GeV} / \mathrm{c}$ (considered with respect to the daughter particle). In the side-band defined by $p_{T}>0.2 \mathrm{GeV} / \mathrm{c}$, we observe 10 events while 10.8 are expected.

Analysing the larger data sample here reported, a new $v_{\tau}$ candidate event was found and presented in June 2012. The decay topology is a three prong vertex, shown in Fig. 3.

The primary vertex is defined in lead by two tracks plus one nuclear fragment. The parent track exhibits a three prong decay topology in the plastic base of the nuclear emulsion, after $1.54 \mathrm{~mm}$ flight length. No nuclear fragments were found at this secondary vertex, which is identified as the $\tau$ decay point. One of the three daughter tracks undergoes a hadronic interaction in emulsion after 10 lead plates: two prongs are associated to four nuclear fragments originating from the tertiary vertex. The second track at the primary vertex is incompatible with the muon hypothesis due to momentum/range considerations. The angle between the tau and the second prong at the primary vertex in the transverse plane is $\phi=168^{\circ}$. Since there are no muons at the primary vertex and the event satisfies all the kinematic selections summarized in Tab. 2, the decay is compatible with being $\tau \rightarrow 3 h$.

The statistical significance of the observation of two $v_{\tau}$ candidate events is currently under evaluation, along with the recomputation of efficiencies and background sources, taking into account the different sample selections. This will be the subject of a forthcoming publication, which will integrate all the improvements and data driven backgrounds described above. The preliminary number of expected $v_{\tau}$ in the analysed data sample is 2.1 , with a preliminary background estimation of 0.2 events.

\section{3}

$v_{\mu} \rightarrow v_{e}$ search

The subdominant $v_{\mu} \rightarrow v_{e}$ oscillation channel is studied thanks to the excellent capabilities of nuclear emulsions to disentangle electrons from photon conversions in identified electromagnetic showers. Here the preliminary results from the analysis of the 5255 interactions contained in the 2008-2009 data will be presented, corresponding to $5.3 \times 10^{19}$ pot. The final analysis will be released in a publication in the near future. 


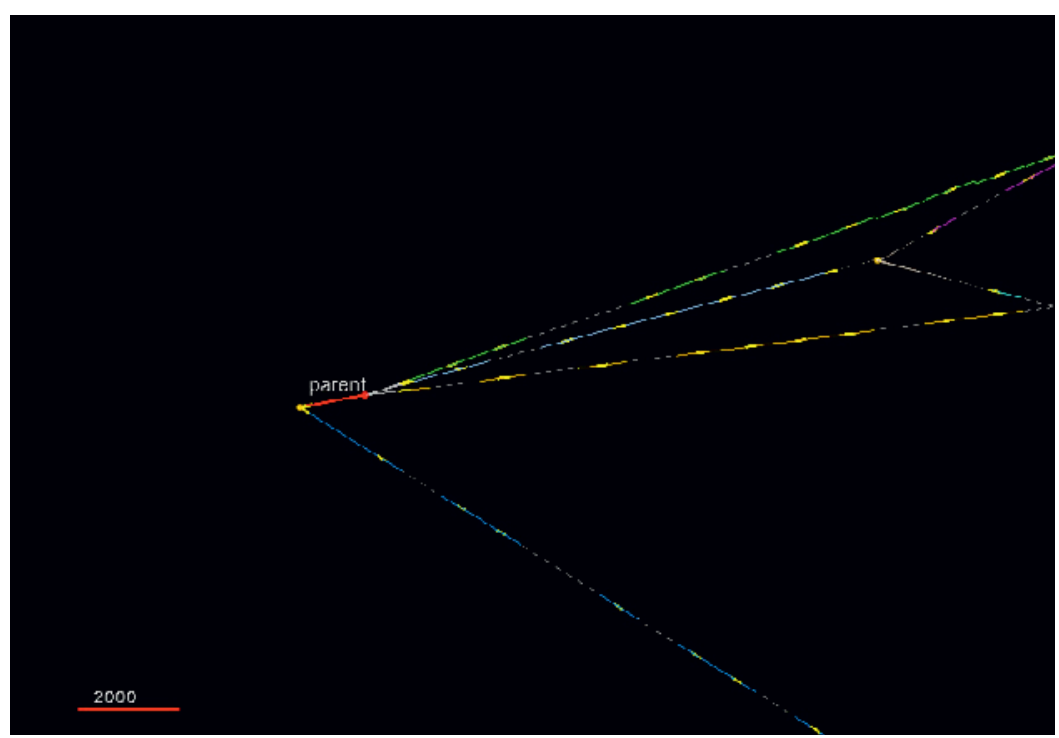

Figure 3. Second tau neutrino candidate event. The $\tau$ parent decays in three prongs $(\tau \rightarrow 3 h$ ), one of them interacts after 10 lead plates.

Table 2. Selection criteria for $v_{\tau}$ candidate definition and corresponding values measured for the second observed tau candidate event.

\begin{tabular}{|c|c|c|c|}
\hline Variable & Cut & Value & Error \\
\hline$\phi$ (tau - hadron) [degree] & $>90$ & 167.8 & \pm 1.1 \\
Average kink angle [mrad] & $<500$ & 87.4 & \pm 1.5 \\
Total momentum at 2ry vertex $[\mathrm{GeV} / \mathrm{c}]$ & $>3.0$ & 8.4 & \pm 1.7 \\
Min invariant mass $\left[\mathrm{GeV} / \mathrm{c}^{2}\right]$ & $0.5<m_{M I N}<2.0$ & 0.96 & \pm 0.13 \\
Invariant mass $\left[\mathrm{GeV} / \mathrm{c}^{2}\right]$ & $0.5<m<2.0$ & 0.80 & \pm 0.12 \\
Transverse momentum at $1 \mathrm{ry}$ vertex $[\mathrm{GeV} / \mathrm{c}]$ & $<1.0$ & 0.31 & \pm 0.11 \\
\hline
\end{tabular}

The $v_{e}$ search is systematically applied on analysed NC-like events, i.e. events without any reconstructed muon $(0 \mu)$. A special procedure is used to select a sample of NC-like events with "shower hints". All the primary tracks are extrapolated to the CS, where it is checked if other tracks with similar direction are present within a predefined distance cut. If 3 or more tracks are found around the primary extrapolation, the event is selected as containing shower hints. An additional volume is open, from the last film of the standard volume up to the most downstream film of the brick. If the automatic algorithm based on a Neural Network reconstruct an electromagnetic shower, and if the primary track initiating the shower is recognized as a single track on the vertex emulsion film, the event is classified as $v_{e}$ candidate event.

Applying the above mentioned procedure, $19 v_{e}$ candidate events were confirmed. 


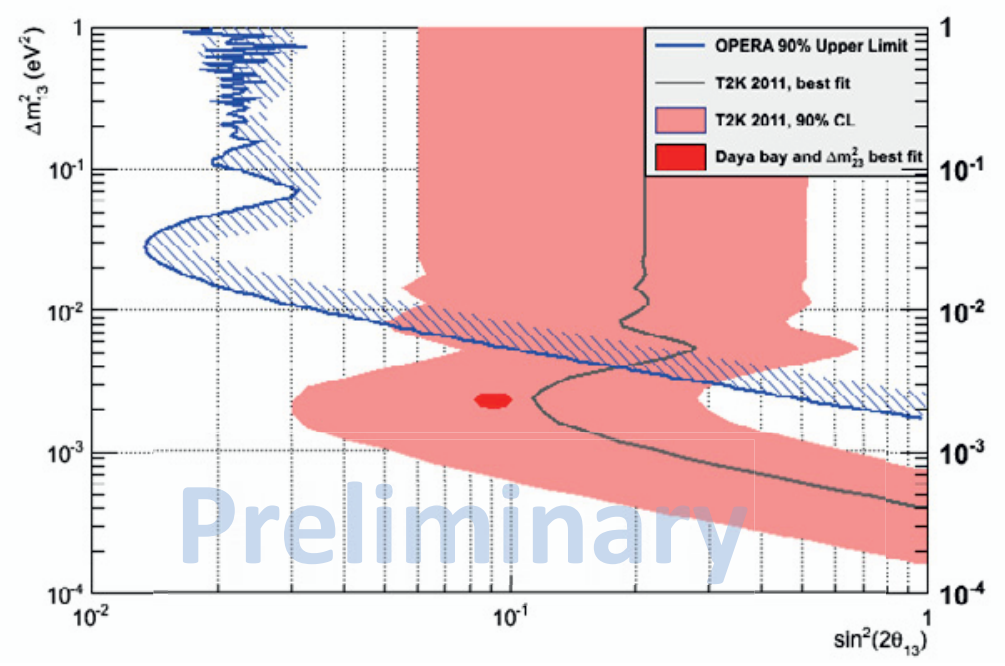

Figure 4. Preliminary limit in the $\left(\Delta m_{13}^{2}, \sin ^{2}\left(2 \theta_{13}\right)\right)$ oscillation parameter space.

We can recognize two background sources mimicking the $v_{e}$ interaction: (1) early photon conversion into an $e^{+} / e^{-}$pair, misidentified as a single electron track, and (2) $\tau \rightarrow e$ decays in $v_{\tau} \mathrm{CC}$ interactions. The first background contribution has been constrained with data driven studies, while the second contribution has been calculated using the up-to-date oscillation parameters. The total background expected in the analysed sample is 0.7 events.

The preliminary number of $v_{e} \mathrm{CC}$ interactions given by the intrinsic beam contamination is evaluated as $n_{e}=20.4 \pm 3.8$. The number of expected beam events is in agreement with our observation, showing no excess signal resulting from $v_{\mu} \rightarrow v_{e}$ oscillations. We can therefore put a limit in the oscillation parameter space excluding the high $\Delta m^{2}$ region $\left(\Delta m^{2}>0.01 \mathrm{eV}^{2}\right)$. The preliminary limit put by OPERA in the $v_{\mu} \rightarrow v_{e}$ parameter space is shown in Fig. 4.

\section{Neutrino velocity measurement}

We will present the updated results of the neutrino velocity measurements using both the standard CNGS data (2009-11 Runs) and the special CNGS "bunched beam" data (Nov 2011 and May 2012).

The key ingredients for the determination of the neutrino velocity are:

- neutrino time-of-flight measurement $\left(\mathrm{ToF}_{v}\right)$, requiring very accurate time distribution and synchronisation between CNGS and OPERA;

- path length measurement, relying on a very precise geodesy campaign over a long baseline.

The measurement procedure is described in detail in Ref. [9]. We briefly recall the principle and the improvements recently brought in the timing uncertainties.

The neutrino start time is defined by the time tagging of the extracted SPS beam. The proton time structure is accurately measured by a fast Beam Current Transformer (BCT) detector (400 MHz bandwidth) read out by a $1 \mathrm{GS} / \mathrm{s}$ Wave Form Digitiser (WFD) with a $250 \mathrm{MHz}$ bandwidth. The 
waveforms recorded for each extraction by the WFD are UTC (Coordinated Universal Time) timestamped with a standard GPS receiver and stored in the CNGS database.

The $\mathrm{ToF}_{v}$ is given by the difference between the arrival time in OPERA and the start time at CERN. At LNGS every millisecond a UTC pulse synchronously derived from the 1PPS of the ESAT2000 GPS system (PPmS) is transmitted from the surface laboratory to the OPERA Master Clock in the underground laboratory via an $8.3 \mathrm{~km}$ long optical fibre. The time base of the Master Clock is transmitted to the OPERA detector sensors, by which the neutrino interaction time is defined.

The required ns accuracy of the relative time tagging at CERN and at the OPERA detector is achieved by adopting two identical systems, composed of a GPS receiver for time-transfer applications operating in "common-view" mode and a Cs atomic clock, installed in both sites. The two systems were calibrated in 2008 by the Swiss Federal Metrology Institute METAS and established a permanent time link at the $2 \mathrm{~ns}$ level between two reference points at CERN and OPERA. This time link was independently verified in 2011 by the German Federal Metrology Institute PTB by taking data at CERN and LNGS with a portable time-transfer device. The difference between the time base of the two GPS receivers was measured to be $(2.3 \pm 1.7)$ ns and subsequently corrected for.

The second fundamental ingredient for the neutrino velocity measurement is the knowledge of the distance between the point where the proton time-structure is measured at CERN and the origin of the OPERA reference frame. The travel path of protons from the BCT to the focal point of the CNGS target is known with millimetric accuracy, $L_{\mathrm{BCT} \text {-target }}=(743.391 \pm 0.002) \mathrm{m}$. When these positions are transformed into the global geodesy reference frame ETRF2000 by relating them to external GPS benchmarks at CERN, they are known within $2 \mathrm{~cm}$ accuracy.

The coordinates of the origin of the OPERA reference frame were measured in 2010 by establishing GPS benchmarks at the two sides of the $10 \mathrm{~km}$ long Gran Sasso highway tunnel and by transporting their positions with a terrestrial traverse down to OPERA. A common analysis in the ETRF2000 of the $3 \mathrm{D}$ coordinates of the OPERA origin and of the target focal point allowed the determination of their distance, $L_{\text {target-OPERA }}=(730534.61 \pm 0.20) \mathrm{m}$. The $20 \mathrm{~cm}$ uncertainty is dominated by the long underground link between the outdoors GPS benchmarks and the benchmark at the OPERA detector.

The baseline considered for the measurement of $\mathrm{ToF}_{v}$ is then the sum of the distance between the CNGS target focal point and the origin of the OPERA detector reference frame, $L_{\text {target-OPERA, and the }}$ distance between the BCT and the focal point, $L_{\mathrm{BCT}-\text { target }}$, i.e. $(731278.0 \pm 0.2) \mathrm{m}$.

\subsection{Recent measurements and installations}

Two instrumental effects were found and corrected for after the first data release.

The first effect is related to the fibre delay from the ESAT2000 to the OPERA Master Clock. The time delay of the $8.3 \mathrm{~km}$ long optical fibre was remeasured in December 2011 using the standard 2-way technique, resulting in a value $73.2 \mathrm{~ns}$ larger than the one determined in 2006 and in 2007. This discrepancy was due to an improper connection of the optical cable reducing the amount of light received by the optical/electrical converter of the Master Clock. The effect of this induced delay, when taken into account in the $\mathrm{ToF}_{v}$ computation, was to artificially underestimate the neutrino arrival time. When proper connections were restored, during a technical intervention, the value of the delay was found to agree with what was measured in 2006 and 2007 within $1 \mathrm{~ns}$ [9].

The second instrumental effect is the mis-calibration of the $10 \mathrm{MHz}$ Vectron OC-050 oscillator disciplining the Master Clock. A test performed in December 2011 and direct measurements during the CNGS winter shutdown revealed that the oscillator frequency was slightly larger $(0.124 \mathrm{ppm})$ than the specifications (Allan deviation of $2 \times 10^{-12}$ over $1 \mathrm{~s}$ ). The OPERA Master Clock is synchronised at every DAQ cycle start $(0.6 \mathrm{~s})$ with the PPmS signal coming from the external GPS. Thus, the time 


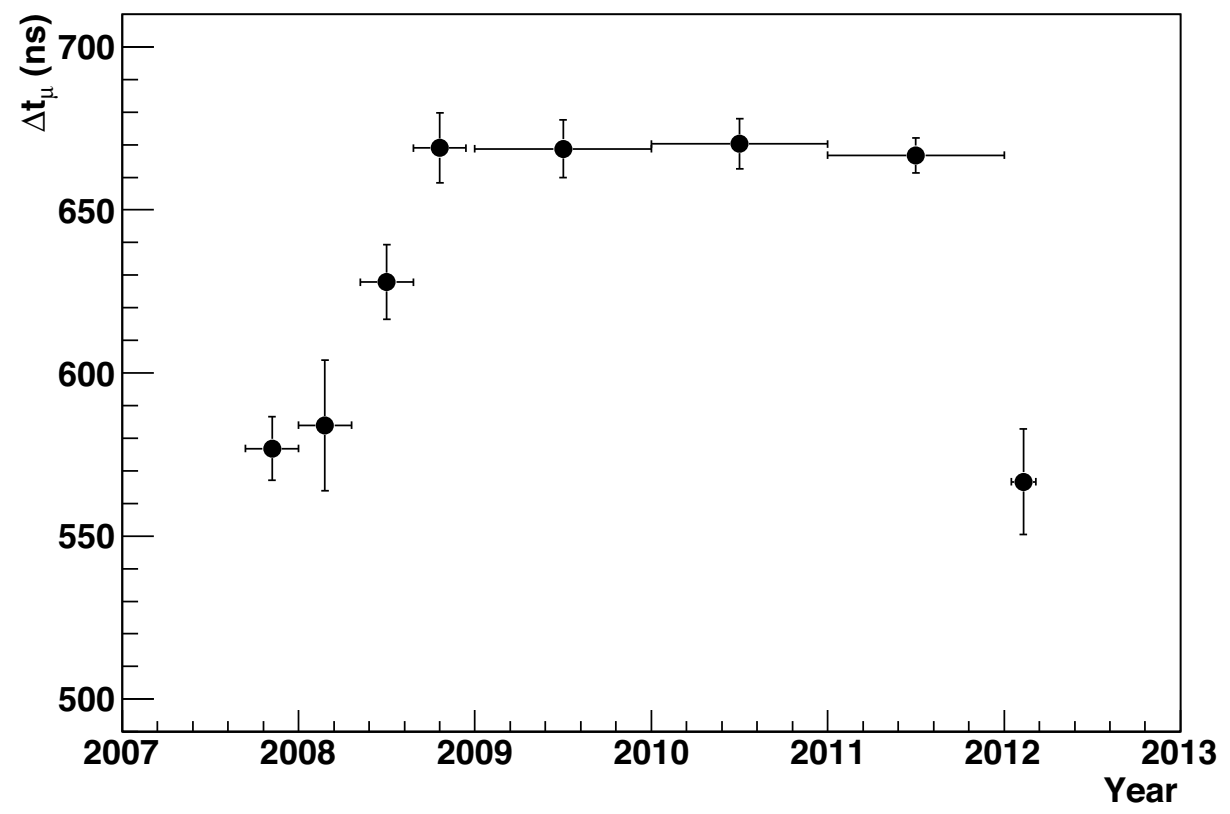

Figure 5. Distribution of the $\Delta t_{\mu}=t_{\mathrm{LVD}}-t_{\mathrm{OPERA}}$. For each year all events are grouped into one single point, but for 2008 they are subdivided into three periods: before May, May-August, and after August.

drift of $124 \mathrm{~ns} / \mathrm{s}$ accumulates in between re-synchronisations occurring every $0.6 \mathrm{~s}$. As a consequence the event time stamp was overestimated by a quantity which depends on the event position inside the DAQ cycle.

The analysis of the OPERA-LVD horizontal cosmic muon coincidences [10] added an additional independent information. The OPERA-LVD data confirm the direct measurements and allow defining when the anomalous conditions of the OPERA timing system occurred and their stability over years. The cosmic muon time of flight over the OPERA-LVD distance $\Delta t_{\mu}$ is plotted versus time in Fig. 5 . There are two abrupt changes in $\Delta t_{\mu}$, one around August 2008 and the other in December 2011. In between $\Delta t_{\mu}$ stays constant thus corresponding to a stable configuration of the detectors' timing systems. The extracted value of $(73.2 \pm 9) \mathrm{ns}$ is compatible with the fibre recalibration described above. The observed $\Delta t_{\mu}$ decrease in December 2011 is related to proper reconnection of the optical fibre to the OPERA Master Clock during a technical intervention. Thus, the anomalous set-up of the OPERA timing system originated in August 2008 and remained in a stable configuration over the whole data taking period considered in the neutrino velocity analysis (2009-2011). By comparing the OPERA time stamps as a function of the time within the OPERA DAQ cycle, a constant time drift of $(114 \pm 14) \mathrm{ns} / \mathrm{s}$ was observed, compatible with the previously reported value of $124 \mathrm{~ns} / \mathrm{s}$. Grouping data year by year, this drift remains constant within the errors.

After all the checks and measurements, some modifications were introduced in the OPERA timing system before the 2012 Run start. The Vectron oscillator was substituted with a new one and a faster optical/electrical converter was inserted in the OPERA Master Clock, in order to avoid the observed problem of time-walk. 
Moreover, a new RPC timing system was installed and used for the analysis of the 2012 CNGS bunched beam data. This new system exploits the signals coming from the Timing Boards (TBs). Seven RPC layers in each magnet are instrumented with TBs (14 TBs per RPC layer), in order to trigger the drift tube detectors. The TB system reconstructs the neutrino interaction time in two steps: the UTC ms component is given by the synchronisation signal (1PPmS) sent by the ESAT LNGS atomic clock, while the sub-ms component is computed as a time difference between the event hits registered by the Timing Board and the front of the $1 \mathrm{PPmS}$ measured by means of a precise TDC specifically installed for the neutrino velocity measurement. Signals from RPCs and the 1PPmS are converted to differential ECL and sent to a CAEN V767 VME TDC module ( $800 \mu$ s range, $0.8 \mathrm{~ns}$ Least Significant Bit and integral non-linearity lower than $0.3 \mathrm{~ns}$ ). To obtain a ns precision over the full range, the TDC has to be calibrated with a ppm precision. For this purpose a thermally stabilized oscillator producing a $5 \mathrm{kHz}$ reference signal sent into a TDC channel has been used during the whole data taking period. In this way, the stability of the TDC oscillator can be monitored event by event, performing also the calibration with the required precision.

Finally, the overall resolution of the timing chain at CERN was recently improved with a new measurement (May 2012) of the BCT-WFD delay $\Delta t_{\mathrm{BCT}}$, the time interval between the transit of protons through the BCT and the arrival of the signal to the WFD. The CERN timing uncertainty is now $\pm 1.8 \mathrm{~ns}$, while the OPERA uncertainty is given separately for the different sub-detectors used for the measurement.

\subsection{Data analysis}

Here we present the updated results on the standard CNGS data from 2009-2011 Runs, the updated results on the special CNGS 2011 bunched beam data [9] and the preliminary results obtained with the 2012 bunched beam data, which will be released in a forthcoming publication.

\subsubsection{CNGS Runs 2009-2011}

The $\mathrm{ToF}_{v}$ measurement of the standard CNGS neutrinos relies on a statistical analysis due to the CNGS extraction duration (see Sec. 1.2). Any proton in the $10.5 \mu$ s long extraction may produce the neutrino detected by OPERA. However, by measuring the time distributions of protons for each extraction for which neutrino interactions are observed in the detector, and summing them together, after proper normalisation one obtains the probability density function (PDF) of the time of emission of the neutrinos within the duration of extraction. The time of a neutrino interaction is defined as that of the earliest hit in the TT. Comparing the time distribution of protons crossing the BCT to the one of neutrino events detected by OPERA allows extracting the $\mathrm{ToF}_{v}$ value. The deviation $\delta t=\mathrm{ToF}_{c}-\mathrm{ToF}_{v}$, i.e. the difference between the ToF of neutrinos and the expected ToF assuming the speed of light, is obtained by an unbinned maximum likelihood analysis of the time tags of the OPERA events with respect to the PDF, as a function of $\delta t$.

The total statistics used for the analysis here presented and reported in Ref. [9] consists of 15223 events (7235 internal, CC and NC, and 7988 external CC), corresponding to about $10^{20}$ protons on target collected during the 2009, 2010 and most of the 2011 CNGS Runs. This allowed estimating $\delta t$ with a small statistical uncertainty, presently comparable to the total systematic uncertainty.

The result of the data analysis shows an arrival time of the neutrinos with respect to the one computed by assuming the speed of light compatible with 0 :

$$
\delta t=\mathrm{ToF}_{\mathrm{c}}-\mathrm{ToF}_{v}=\left(6.5 \pm 7.4(\text { stat. })_{-8.0}^{+8.3}(\text { syst. })\right) \mathrm{ns}
$$


There is furthermore no indication for a dependence on the period of the year, time of the day, neutrino energy, beam intensity or containment in the detector [9]. The relative difference of the muon neutrino velocity with respect to the speed of light is:

$$
(v-c / c)=\left(2.7 \pm 3.1(\text { stat. })_{-3.3}^{+3.4}(\text { syst. })\right) \times 10^{-6}
$$

\subsubsection{CNGS bunched beam 2011}

In order to exclude possible systematic effects related to the statistical extraction of the time of flight, the $\mathrm{ToF}_{v}$ measurement was performed with a dedicated CNGS beam. The modified SPS super-cycle consisted of a single extraction including four bunches about 3 ns long (FWHM) separated by 524 $\mathrm{ns}$, yielding a total of $1.1 \times 10^{12}$ protons per cycle. This narrow-bunch wide-spacing neutrino beam allowed performing time of flight measurements at the single event level.

During the CNGS 2011 bunched beam (from October 22 to November 6, 2011), 6 internal and 14 external events were collected and retained after selection. Given the short bunch length and the relatively long inter-bunch distance one could unambiguously associate each neutrino event to its corresponding proton bunch. The measurement at the single interaction level with the TT is $\delta t=(-1.9 \pm 3.7 \text { (stat. })_{-8.0}^{+8.3}$ (syst.)) ns, in agreement with the standard CNGS data analysis. At first order, systematic uncertainties related to the bunched beam operation are equal or smaller than those affecting the result with the nominal CNGS beam.

This result largely excludes possible biases affecting the statistical analysis based on the proton PDF. It also indicates the absence of significant biases due to the cumulative response of the beam line to long proton pulses, as well as pulse duration effects in the BCT response.

The same measurement was obtained using the RPC timing information for CC events with a muon crossing the spectrometer. Every RPC plane provides an independent time measurement along each muon track at a known position along the detector. Averaging all the muon time stamps, the value of $\delta t$ obtained from the RPC data is: $\delta t=(-0.8 \pm 3.5 \text { (stat. })_{-9.6}^{+9.9}$ (syst.)) ns.

\subsubsection{CNGS bunched beam 2012}

Between the 10th and 24th of May 2012 CERN provided a new dedicated neutrino velocity run. In the new 2012 narrow-bunch short-spacing beam (BB) the bunch separation is only $100 \mathrm{~ns}$ in order to increase the number of delivered neutrinos per time unit. In each CNGS cycle lasting $13.2 \mathrm{~s}$ one single extraction delivers 4 batches of 16 proton bunches. Each $2.8 \mathrm{~ns}$ long (RMS) bunch has $\sim 10^{11}$ protons providing an intensity by a factor 6 higher than during the $2011 \mathrm{BB}$ run.

In two weeks of run a total of $1.8 \times 10^{17}$ pots were delivered. OPERA recorded 104 on-time events, 67 involving TT detectors and 62 involving RPC detectors. Independent analyses were performed for events involving TT and RPC detectors.

The 67 on-time events with TT hit information were selected using the same procedures described in Ref. [9]. The retained data sample is composed of 59 events. The neutrino interaction time was computed using two methods. The first method (Method 1) relies on the information of the earliest hit as described in Ref. [9]. The second method (Method 2) exploits the time information of all hits of the 3D muon track. Using this method, $48 \mathrm{CC}$ events have been selected. The second method results in a better statistical accuracy due to the larger number of information coming from the whole track. However, the second method misses NC-like events. In the upper left (right) plot of Fig. 6 the $\delta t=$ $\mathrm{ToF}_{c}-\mathrm{ToF}_{v}$ is shown for the first (second) method respectively. The final result is reported in Tab. 3. 
For the RPC analysis (Method 3), only neutrino interactions producing a clear muon track were used. The total number of events used by this analysis is 58. The $\delta t$ distribution is shown in Fig. 6 and the final result is given in Tab. 3 .

The RPC-TB hits acquired by the VME TDC are selected and then corrected by the known calibrated delays (for the position along the read-out strip, information from OPERA DAQ is used) and averaged. The time resolution using the RPC-TB setup was computed using muons crossing both spectrometers. The value is $\sigma_{t}=1.1 \mathrm{~ns}$, which is well below the RMS of the distribution shown in Fig. 6 (Method 4). The final $\delta t$ is given in Tab. 3.

In summary, four different non-independent results were obtained for the neutrino velocity measurements. Measurements 1 and 2 are statistically correlated since they share the same DAQ system and detector. They are also correlated with measurement 3 since they share the same DAQ system. Measurement 4 is mostly uncorrelated with the others having a different timing system. The residual correlation is on the systematic error of the common part of the timing chain, namely the delays from BCT up to the entrance in the underground hall.

In order to obtain a single combined result Methods 2 and 4 were used since they provide the smallest statistical and systematic errors and - moreover - they are almost completely uncorrelated thus providing maximum information. Values obtained with Method 1 were used for events when Method 2 could not be used because of the absence of muon track (20 events).

For the events where the muon is crossing at least one spectrometer the momentum and the charge of the muon is measured. Profiting of the charge information, results are given separately for $v_{\mu}$ and $\bar{v}_{\mu}$ present in the CNGS beam ( $\sim 2 \%$ contamination, see Sec. 1.2$)$. In 3 out of 58 CC events, the muon charge is found to be positive.

The final result, separating the $v$ and $\bar{v}$ contributions, is:

$$
\left.\delta t_{v}=(0.6 \pm 0.4 \text { (stat.) } \pm 3.0 \text { (syst. })\right) \mathrm{ns}
$$

and:

$$
\delta t_{\bar{v}}=(1.8 \pm 1.4 \text { (stat.) } \pm 3.2 \text { (syst.)) ns }
$$

respectively. It has been assumed that all the NC-like events result from $v$ interactions. Since both results are compatible with zero a limit on the deviation from the speed of light was derived:

$$
-1.8 \times 10^{-6}<\left(v_{v}-c\right) / c<2.3 \times 10^{-6}
$$

and:

$$
-1.6 \times 10^{-6}<\left(v_{\bar{v}}-c\right) / c<3.1 \times 10^{-6}
$$

for $v$ and $\bar{v}$ respectively (the slight difference on the systematic errors of the two above measurements comes from the different contributions of each method to the systematic error).

Table 3. Separate results for $v+\bar{v}$ for each of the four methods reported in the text. All reported time values are in ns.

\begin{tabular}{|c|c|c|c|c|}
\hline Method & Events & $\delta t$ & Stat. & Syst. \\
\hline 1 & 59 & -2.1 & 1.1 & 4.4 \\
2 & 48 & 1.2 & 1.0 & 3.3 \\
3 & 58 & -2.5 & 1.8 & 5.3 \\
4 & 49 & 0.6 & 0.4 & 3.6 \\
\hline
\end{tabular}



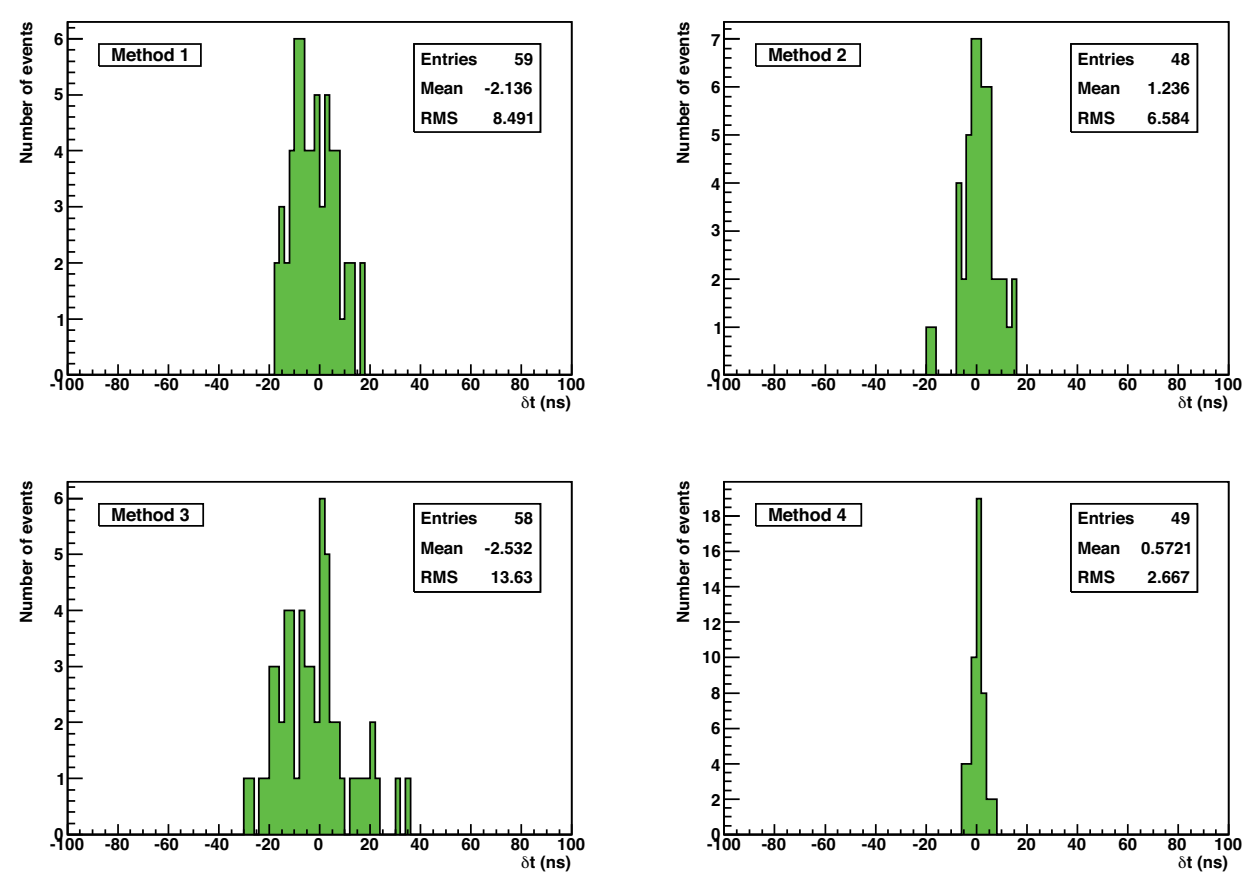

Figure 6. Neutrino time distribution for events selected for the TT analysis using the first hit (Method 1), for the TT analysis using all muon hits (Method 2), for events selected for the RPC analysis using all muon hits (Method 3) and for events selected for the RPC analysis using the trigger boards (Method 4).

\section{Conclusions}

The OPERA experiment, aiming at the first detection of neutrino oscillations in appearance mode, is successfully collecting CNGS data since 2008. Two $v_{\tau}$ candidate events were observed in the decay search of 2008-2009 and part of 2010-2011 CNGS data. The significance of the observation is currently under evaluation, the preliminary number of expected $v_{\tau}$ events is 2.1 with 0.2 expected background events.

Regarding the $v_{\mu} \rightarrow v_{e}$ oscillation search, $19 v_{e}$ candidate events were identified analysing the 2008-2009 data. Since there is no $v_{e}$ excess signal, OPERA can put a limit in the parameter space excluding the high $\Delta m^{2}$ region.

By the end of the 2012 Run, the fifth year of data taking, OPERA should almost reach the nominal CNGS exposure. The $\tau$ search and the subdominant $v_{\mu} \rightarrow v_{e}$ oscillation search are being pursued, publications on both subjects are going to be released in the near future.

Finally, OPERA provided a precision measurement of the neutrino velocity over the $730 \mathrm{~km}$ CNGS baseline. The sensitivity of the measurement of $(v-c) / c$ is about one order of magnitude better than former accelerator neutrino experiments. The OPERA experiment, after correcting and improving its timing system, has confirmed its results obtained from 2009-2011 standard CNGS data 
and from the special 2011 bunched beam data [9] with a new measurement in May 2012, clearly showing no significant difference between the neutrino/antineutrino velocity and the speed of light.

\section{References}

[1] R. Acquafredda et al. (OPERA Collaboration), JINST 4, (2009) P040018.

[2] N. Agafonova et al. (OPERA Collaboration), New J. Phys. 14, (2012) 013026.

[3] A. Anokhina et al. (OPERA Collaboration), JINST 3, (2008) P07005.

[4] N. Agafonova et al. (OPERA Collaboration), New J. Phys. 14, (2012) 033017.

[5] N. Armenise et al., Nucl. Instrum. Methods A 551, (2005) 261;

M. De Serio et al., Nucl. Instrum. Methods A 554, (2005) 247;

L. Arrabito et al., Nucl. Instrum. Methods A 568, (2006) 578;

K. Moroshima and T. Nakano, JINST 5, (2010) P04011.

[6] N. Agafonova et al. (OPERA Collaboration), New J. Phys. 13, (2011) 053051.

[7] N. Agafonova et al. (OPERA Collaboration), Phys. Lett. B 961, (2010) 138.

[8] A. Kayis-Topasku et al. (CHORUS Collaboration), New J. Phys. 13, (2011) 093002.

[9] T. Adam et al. (OPERA Collaboration), JHEP 10, (2012) 093.

[10] N.Y. Agafonova et al. (LVD and OPERA Collaborations), Eur. Phys. J. Plus 127, (2012) 71. 